\title{
Tuberculose em 228 casos de autópsias
}

\section{Tuberculosis in 228 cases of autopsies}

Priscilla Mariana Freitas Aguiar Feitosa ${ }^{1,2}$. Márcia Valéria Pitombeira Ferreira ${ }^{1,2}$. Renan Siebra Capibaribe Silveira ${ }^{1}$. Thamiris Silva de Queiroz ${ }^{1}$. Lily Ferreira Aguiar ${ }^{3}$. Letícia Neves Solon Carvalho ${ }^{4}$.

1 Universidade Federal do Ceará (UFC), Fortaleza, Ceará, Brasil. 2 Hospital Universitário Walter Cantídio (HUWC), Fortaleza, Ceará, Brasil. 3 Universidade de Fortaleza (UNIFOR), Fortaleza, Ceará, Brasil. 4 Universidade de São Paulo (USP), São Paulo, São Paulo, Brasil

\section{RESUMO}

Objetivos: tuberculose é a principal causa de morte por um único agente infeccioso no mundo e há poucos trabalhos com autópsia analisando seus aspectos anatomopatológicos. Dessa forma, o objetivo do artigo é analisar características anatomopatológicas de pacientes diagnosticados com tuberculose em autópsia e sua relação com aspectos epidemiológicos e clínicos. Metodologia: estudo longitudinal, retrospectivo, descritivo dos dados dos arquivos de registros e dos arquivos de laudos dos casos de tuberculose do serviço de autópsia do Departamento de Patologia da Universidade Federal do Ceará entre 1959 e 2015 . Resultados: foram 7236 autópsias com 228 casos de tuberculose (3,2\%). Em 39\% não houve suspeita clinicamente de tuberculose e $16 \%$ foram suspeitos. Predominou sexo masculino e a média de idade de 34,4 anos. As doenças mais associadas foram desnutrição (21,1 \%), Síndrome da Imunodeficiência Adquirida (SIDA) (16,9\%), câncer (11,3\%), cirrose hepática (7,0\%). Tuberculose disseminada ocorreu em 53,5\%, pulmonar em $35,1 \%$ e extrapulmonar em $11,4 \%$, destes, o sistema nervoso central foi o mais afetado com meningite (46,2\%) e meningoencefalite $(23,1 \%)$. Conclusões: Tuberculose não foi clinicamente diagnosticada em uma quantidade importante de casos. Faixa etária mais jovem e mais casos nas décadas de 80 e 90 revelou a importante associação entre SIDA e tuberculose. A tuberculose teve apresentação variada, complexa e grave.

Palavras-chave: Tuberculose. Autópsia. Doença infecciosa. Doenças infectocontagiosas.

\section{ABSTRACT}

Objectives: Tuberculosis is the main cause of death by a single infectious agent in the world. However, few studies have been published about the anatomopathological aspects of tuberculosis (TB) in the organism as a whole. The objective of this article is to analyze the anatomopathological characteristics of deceased patients diagnosed with tuberculosis revealed by autopsy and their relationship with epidemiological and clinical aspects. Methodology: Longitudinal, retrospective, descriptive study of data from records files and reports files of tuberculosis cases from the autopsy at the pathology service of Universidade Federal do Ceará between 1959 and 2015. Results: There were 7236 autopsies with 228 cases of tuberculosis (3.2\%). In 39\% there was no clinical suspicion of tuberculosis and 16\% were suspected. Male gender predominated and an average age of 34.4 years. The most associated diseases were malnutrition (21.1\%), Acquired Immunodeficiency Syndrome (AIDS) (16.9\%), cancer (11.3\%), liver cirrhosis (7.0\%). Disseminated tuberculosis occurred in $53.5 \%$, pulmonary in $35.1 \%$ and extrapulmonary in $11.4 \%$, the central nervous system was the most affected with meningitis (46.2\%) and meningoencephalite (23.1\%). Conclusions: Tuberculosis has not been clinically diagnosed in a significant number of cases. Younger age group and more cases in the 1980s and 90s revealed an important association between AIDS and Tuberculosis. Tuberculosis had a varied, complex and severe presentation.

Keywords: Tuberculosis. Autopsy. Infectious diseases. Transmissible diseases.

Autor correspondente: Priscilla Mariana Freitas Aguiar Feitosa, Rua Ana Bilhar, 54, Meireles, Fortaleza, Ceará. CEP: 60160-110. Telefone: +55 85 98541-0726. E-mail: pripiaguiar@hotmail.com

Conflito de interesses: Não há qualquer conflito de interesses por parte de qualquer um dos autores.

Recebido em: 24 Jan 2021; Revisado em: 02 Ago 2021; Aceito em: 03 Nov 2021. 


\section{INTRODUÇÃO}

A tuberculose (TB) continua como um grave problema de saúde pública. Sob controle e praticamente extinta, ressurgiu nos anos 80 em decorrência de migrações dos países de alta incidência, a pobreza e a marginalização social, o desinvestimento nos programas de controle e a infecção pelo Vírus da Imunodeficiência Humana (HIV). ${ }^{1}$

Os sinais, sintomas e as manifestações radiológicas dependem do tipo de apresentação da TB. Classicamente, as principais formas de apresentação são a forma primária, a pós-primária (ou secundária) e a miliar. Os sintomas clássicos, como tosse persistente seca ou produtiva, febre vespertina, sudorese noturna e emagrecimento, podem ocorrer em qualquer das três apresentações.

Em 2018, 10 milhões de pessoas adoeceram com tuberculose em todo o mundo, sendo 5,7 milhões de homens, 3,2 milhões de mulheres e 1,1 milhão de crianças. Houve casos em todos os países e faixas etárias. Apenas 30 países representaram $87 \%$ dos novos casos de TB. Índia lidera a contagem, seguida por China, Indonésia, Filipinas, Paquistão, Nigéria, Bangladesh e África do Sul e esses países juntos são responsáveis por dois terços do total no mundo e as Américas representam 2,9\%. ${ }^{2}$ De acordo com a nova classificação da OMS 2016-2020, o Brasil ocupa a $20^{\mathrm{a}}$ posição na lista dos 30 países prioritários para TB e a $19^{\mathrm{a}}$ posição na lista dos 30 países prioritários para TB-HIV. ${ }^{3}$

Um total de 1,5 milhão de pessoas morreram por tuberculose em 2018 sendo 251 mil pessoas coinfectadas com HIV. Em todo o mundo a TB é uma das 10 principais causas de morte e a principal causa de morte por doença infecciosa superando as mortes por HIV/AIDS. ${ }^{2}$

Estudos de necropsia para analisar achados anatomopatológicos na TB têm papel importante para maior caracterização da doença, sua relação com as comorbidades e o impacto na saúde da comunidade local e global. Em anos recentes países com moderada a alta incidência de tuberculose tem realizado publicações sobre TB em autópsias como África, Índia e China. ${ }^{4}$
O objetivo deste trabalho foi analisar características anatomopatológicas de pacientes diagnosticados com tuberculose em autópsia e sua relação com alguns aspectos epidemiológicos e clínicos; além de fazer um resgate histológico da TB e determinar a frequência de TB nas autópsias realizadas no Departamento de Patologia e Medicina Legal da Faculdade de Medicina da Universidade Federal do Ceará (DPML/FAMED/UFC) no período de 1959 a 2015.

\section{MATERIAL E MÉTODOS}

O estudo foi aprovado pelo Comitê de Ética em Pesquisa da Instituição com parecer número 1.198.865.

Estudo longitudinal, retrospectivo, descritivo, baseado em levantamentos de dados dos arquivos de registros e dos arquivos de laudos do serviço de autópsia dos casos com diagnóstico de tuberculose no período de 1959 a 2015 no Departamento de Patologia e Medicina Legal da Faculdade de Medicina da Universidade Federal do Ceará (DPML/FAMED/ UFC). Os dados foram armazenados e analisados no software Microsoft Excel 2013.

\section{RESULTADOS}

De 1959 a 2015 foram 7236 autópsias no DPML/FAMED/ UFC dos quais 228 casos tiveram o diagnóstico de tuberculose (3,2\%), sendo 144 pacientes masculinos $(63,2 \%)$ e 84 femininos (36.8\%). A faixa de idade de 20 a 39 anos foi a mais afetada tanto em homens quanto mulheres. A média de idade foi de 34,4 anos. Entre os homens a média foi de 35,4 anos e mulheres foi de 32,4 anos (Tabela 1). Os maiores números de casos ocorreram nas décadas de 80 e 90, com 89 casos (39\%) e 56 casos (25\%), respectivamente. Em 103 casos (45\%) havia o diagnóstico clínico de TB, em 89 casos (39\%) tuberculose não foi pensada clinicamente e em 36 casos (16\%) a TB foi suspeitada clinicamente.

Tabela 1. Relação entre faixa etária e sexo em 228 pacientes com diagnóstico de tuberculose em autópsia no DPML/ FAMED/UFC de 1959 - 2015.

\begin{tabular}{lccc}
\hline \multicolumn{1}{c}{ Faixa etária (anos) } & Masculino (144) & Feminino (84) & Total (228) \\
\hline$<\mathbf{1}$ & $5(3,4 \%)$ & $2(2,3 \%)$ & $7(3,0 \%)$ \\
$\mathbf{1}$ a $\mathbf{4}$ & $7(4,8 \%)$ & $8(9,5 \%)$ & $15(6,5 \%)$ \\
$\mathbf{4}$ a $\mathbf{1 4}$ & $8(5,5 \%)$ & $7(8,3 \%)$ & $15(6,5 \%)$ \\
$\mathbf{1 5}$ a $\mathbf{1 9}$ & $4(2,7 \%)$ & $2(2,3 \%)$ & $6(2,6 \%)$ \\
$\mathbf{2 0}$ a $\mathbf{2 9}$ & $24(16,6 \%)$ & $15(17,8 \%)$ & $39(17,1 \%)$ \\
$\mathbf{3 0} \mathbf{3 9}$ & $23(15,9 \%)$ & $13(15,4 \%)$ & $36(15,7 \%)$ \\
$\mathbf{4 0}$ a 49 & $15(10,4 \%)$ & $9(10,7 \%)$ & $24(10,5 \%)$ \\
$\mathbf{5 0} \mathbf{5 9}$ & $17(11,8 \%)$ & $4(4,7 \%)$ & $21(9,2 \%)$ \\
$>\mathbf{6 0}$ & $15(10,4 \%)$ & $10(11,9 \%)$ & $25(10,9 \%)$ \\
Sem informação & $26(18,0 \%)$ & $14(16,6 \%)$ & $40(17,5 \%)$ \\
\hline
\end{tabular}


Em 157 casos (69\%), tuberculose ativa foi o diagnóstico principal sem doença de base clinicamente associada ou revelada na autópsia. Dos 228 casos, $122(53,5 \%)$ apresentaram TB disseminada, $80(35,1 \%)$ TB pulmonar e $26(11,4 \%)$ TB extra-pulmonar. Desta última 46,2\% (12) com diagnóstico de TB meníngea; 23,1\% (6) TB meningoencefálica; 19,2\% (5) TB ganglionar, 3,8\% (1) TB articular; 3,8\% (1) TB peritoneal e $3,8 \%$ (1) TB renal (Tabela 2).

Tabela 2. Tuberculose em 228 pacientes em autópsia no DPML/ FAMED/UFC de 1959 - 2015

\begin{tabular}{llll}
\hline Disseminada & Pulmonar & Extrapulmonar & N (\%) \\
$\mathbf{1 2 2} \mathbf{( 5 3 , 5 \% )}$ & $\mathbf{8 0 ( 3 5 . 1 \% )}$ & \multicolumn{1}{c|}{$\mathbf{2 6}(\mathbf{1 1}, \mathbf{4} \%)$} & \\
\hline & & Meníngea & $12(46,2 \%)$ \\
& Meningoencefálica & $6(23,1 \%)$ \\
& Ganglionar & $5(19,2 \%)$ \\
& Articular & $1(3,8 \%)$ \\
& Peritoneal & $1(3,8 \%)$ \\
& & Renal & $1(3,8 \%)$ \\
\hline
\end{tabular}

Observou-se que $156(68 \%)$ dos pacientes apresentaram alguma condição associada a TB, sendo 85 (37\%) complicações agudas, 71 (31\%) doenças crônicas de base. Das condições crônicas foram observadas: desnutrição $(21,1 \%)$, síndrome da imunodeficiência adquirida (SIDA) (16,9 \%), câncer (11,3 \%), cirrose hepática $(7,0 \%)$, insuficiência renal crônica (IRC) $(5,6$ $\%)$, calazar $(5,6 \%)$, hepatite $(5,6 \%)$, insuficiência cardíaca congestiva (ICC) $(5,6 \%)$, lúpus $(5,6 \%)$, doença pulmonar obstrutiva crônica (DPOC) (4,2\%), alcoolismo (2,8 \%), doença de Crohn (2,8\%), diabetes mellitus (DM) (2,8\%) e hanseníase $(2,8 \%)$. Das complicações agudas, a insuficiência respiratória, septicemia, broncopneumonia e choque hipovolêmico foram as mais frequentes, tendo alguns dos pacientes mais de uma complicação aguda, sendo a insuficiência respiratória o evento que mais se repetiu.

\section{DISCUSSÃO}

Autópsia possibilita o diagnóstico de doenças não suspeitadas ou elucidadas antes da morte. ${ }^{5}$ Nosso trabalho evidenciou que $39 \%$ dos casos não teve tuberculose como diagnóstico clínico e $16 \%$ dos casos suspeitos clinicamente foram prontamente confirmados pela autópsia. Tivemos 3,2\% de tuberculose de todas as autópsias do nosso serviço. A tuberculose clinicamente não diagnosticada compõe uma proporção substancial de casos ativos de TB diagnosticados na autópsia, com porcentagens até maiores a encontradas por nós. ${ }^{6}$ Tuberculose ativa foi o diagnóstico principal em $69 \%$ dos nossos casos. Joaquim, A. F. et al, evidenciou que TB era a doença principal em $49 \%$ dos casos. ${ }^{5}$ Enquanto a tuberculose como doença principal foi observada em 28,38\% dos casos na publicação de Sangma, M.M. et al, 2014. Nos achados de Garg, M. et al, 2011, tuberculose como doença principal foi $30 \%$ dos pacientes. ${ }^{7}$ Tivemos uma quantidade elevada de casos com diagnóstico de TB como diagnóstico principal. Isto pode refletir dados clínicos não informados nas requisições de autópsia ou não investigação clínica de doenças associadas, ou a gravidade dos nossos casos pode ter interferido no tempo de avaliação clínica.

Analisando-se as características dos indivíduos, os resultados do presente estudo são concordantes com a literatura que relatam a maior prevalência e proporção ocorrendo na população masculina. ${ }^{8}$

No Brasil 85\% dos casos de TB ocorrem em maiores de idade variando de 35 a 44 anos. $^{7}$ Estudos anteriores mostram que óbitos por TB foram maiores em pacientes jovens, uma possível explicação é que as pessoas com mais de 60 anos foram menos afetadas pela pandemia do HIV. ${ }^{9}$ A maioria dos nossos casos mostrou acometimento na faixa de 20 a 39 anos de idade com média geral de 34,4 anos. Nosso trabalho evidenciou que a maioria dos casos autopsiados com diagnóstico de TB ocorreu nas décadas de 80 e 90, momento mundialmente relacionado ao advento da AIDS.

Na nossa casuística, a desnutrição e a SIDA lideraram a lista de condições crônicas associadas. Outras associações observadas foram neoplasia, cirrose hepática, insuficiência renal crônica, calazar, hepatite, insuficiência cardíaca congestiva, lúpus, DPOC, alcoolismo, doença de Crohn, diabetes mellitus e hanseníase.

A desnutrição e a TB foi observada em 21,1\% dos nossos pacientes. Carrazedo, et al refere que os principais fatores de risco para TB incluem o indivíduo ser natural de uma região de alta prevalência, possuir um sistema imune deprimido, podendo ser secundário a desnutrição ou vacinação recente com vírus vivos, e um baixo nível socioeconómico. ${ }^{10}$

A TB é uma infecção oportunista maior em indivíduos infectados pelo HIV, sendo, frequentemente, a forma definidora de SIDA; e a primeira advertência para a imunodeficiência. Globalmente, a TB é a maior causa de morte em indivíduos infectados pelo HIV, o HIV é um dos maiores impulsores da epidemia de TB. ${ }^{1}$ Carrazedo, A.M.V., et al. observou em relação TB/HIV que 13,6\% eram HIV positivos, sendo que 53\% tiveram TB como doença indicadora de SIDA, reafirmando a TB como responsável por uma taxa de mortalidade média de $30 \%$ entre os indivíduos com HIV/SIDA. Encontramos 16,9\% de casos clinicamente diagnosticados com SIDA e que à autópsia apresentaram TB, revelando a importante associação entre essas doenças.

Dos casos de TB em todo o mundo, aproximadamente $15 \%$, podem estar ligados ao diabetes mellitus (DM), ${ }^{11}$ já em nossa casuísta, a associação TB e DM representou 2,8 \%². Esse dado pode estar associado ao tipo da amostragem ou à não informação clínica na requisição de autópsia.

Associação entre álcool e TB nos nossos resultados foi de $2,8 \%$. Relação entre consumo de álcool e a tuberculose é longamente conhecida. ${ }^{10} \mathrm{~A}$ informação sobre o consumo de álcool nem sempre é revelada pelo paciente o que pode explicar nosso resultado. 
A frequência da TB aumenta significativamente com o aumento da imunossupressão, assim como aumentam as formas extrapulmonares e disseminada. ${ }^{1}$ Dos 7,0 milhões de casos novos e de recaídas notificados em 2018, 5,9 milhões (85\%) tinha TB pulmonar. A TB extrapulmonar representou 15\% dos 7,0 milhões de casos notificados em 2018, variando de $8 \%$ na Região do Pacífico Ocidental a 24\% na Região do Mediterrâneo Oriental. ${ }^{2}$ A TB disseminada ou miliar representou em nossa casuística a maioria dos casos $(53,5 \%)$, seguida por TB pulmonar $(35,1 \%)$ e TB extrapulmonar $(11,4 \%)$. Nos estudos de autópsia em adultos, a TB miliar foi documentada em maior proporção de pacientes, representando $0,3 \%-13,3 \%$ de todas as autópsias e $11,9 \%-40,5 \%$ de todos os casos de TB. ${ }^{12} \mathrm{~A}$ TB disseminada, caracterizada por afetar dois ou mais órgãos, anteriormente observada quase exclusivamente em crianças ou em pessoas com supressão imunológica, está sendo cada vez mais encontrada em adultos sem defeito imunológico aparente. A maioria desses pacientes são imigrantes em países com incidência moderada a alta de TB, onde a barreira linguística e vários fatores dificultam o acesso à moradia, emprego, sistema de saúde, o que pode atrasar o diagnóstico e, assim, facilitar o avanço da doença. ${ }^{13}$ Em nossos resultados, o sistema nervoso central foi o mais afetado foco de TB extrapulmonar com $46,2 \%$ de meningite tuberculosa e $23,1 \%$ de meningoencefalite tuberculosa. Considerando que os casos foram de pacientes autopsiados, revelou a forma mais grave da tuberculose. Bavikar, R. et al, 2015, encontrou a meningite tuberculosa como a forma extrapulmonar mais comum em pacientes autopsiados. ${ }^{14} \mathrm{~A}$ doença afeta o sistema nervoso central, particularmente meninges, causando assim alta taxa de mortalidade. Aproximadamente $1 \%$ de todos os casos de TB ativa e 5-10\% dos casos de tuberculose extrapulmonar tem meningite tuberculosa. É mais comum em crianças e pacientes infectados pelo HIV. ${ }^{15}$

A tuberculose ganglionar foi o terceiro sítio mais afetado com $19,2 \%$ na nossa casuística. TB ganglionar continua sendo a forma mais comum de tuberculose extrapulmonar nos países em desenvolvimento e afeta mais as crianças e adultos jovens. ${ }^{15}$

A tuberculose articular, peritoneal e renal teve a mesma proporção de $3,8 \%$ de TB extrapulmonar nos nossos casos. A TB renal é a segunda forma mais comum de TB extrapulmonar e considerada como subparte do TB do trato genitourinário.

\section{REFERÊNCIAS}

1. Harries AD. Robert Koch and the discovery of the tubercle bacillus: the challenge of HIV and tuberculosis 125 years later. Int J Tuberc Lung Dis. 2008;12(3):241-9.

2. World Health Organization. Tuberculosis[Internet]. 2020. [cited 2020 Oct. 06]. Available from: https://www.who.int/news-room/ fact-sheets/detail/tuberculosis

3. Brasil. Ministério da Saúde. Secretaria de Vigilância em Saúde. Manual de Recomendações para o Controle da Tuberculose no Brasil [Internet]. Brasília: Ministério da Saúde; 2019 [cited 2020 Oct.
A tuberculose peritoneal faz parte da TB extrapulmonar abdominal e representa o sexto local mais comum da TB extrapulmonar. A TB osteoarticular representa $2-5 \%$ de toda a TB e $11-15 \%$ de TB extrapulmonar, com a coluna vertebral sendo o local mais afetado. ${ }^{15}$

Nosso trabalho também evidenciou complicações agudas associadas à tuberculose, sendo muitas destas responsáveis pela causa da morte como a insuficiência respiratória, septicemia, broncopneumonia e choque hipovolêmico.

\section{CONCLUSÃO}

Os resultados mostraram que a TB é uma doença que deve ser investigada na prática clínica visto que não foi considerada em $39 \%$ dos casos autopsiados e foi confirmada em $16 \%$ dos casos suspeitos de tuberculose.

A faixa etária mais jovem de acometimento dos pacientes e o maior número de casos de tuberculose autopsiados no serviço terem ocorrido nas décadas de 80 e 90 revelou a importante associação entre SIDA e TB.

O trabalho mostrou que a tuberculose teve uma apresentação variada, complexa e grave, com achado de TB disseminada como a forma clínica mais frequente e o acometimento do sistema nervoso central como o mais incidente na forma extrapulmonar.

Muitos trabalhos sobre tuberculose em autópsia eram relacionados à AIDS e recentemente a achados de TB em autópsias forenses. Vem crescendo o número de publicações sobre tuberculose em casos autopsiados do ponto de vista da anatomia patológica geral e aplicada a técnicas sofisticadas de diagnóstico de TB. Essas abordagens poderão orientar gestores e profissionais de saúde para eliminar a ocorrência de óbitos evitáveis e injustos por tuberculose e compreender melhor a patogenia e novas formas diagnósticas e terapêuticas.

No combate a uma doença com alta incidência e mortalidade global é necessário ter uma compreensão conceitual e clara da TB de uma forma geral para somar esforços coletivos entre pesquisadores, profissionais da saúde pública e da atenção primária, bem como da população, ampliando os conhecimentos na tentativa de reduzir uma doença sem fronteiras e de relevância significativa na prática clínica.

06]. Available from: https://bvsms.saude.gov.br/bvs/publicacoes/ manual_recomendacoes_controle_tuberculose_brasil_2_ed.pdf

4. Sangma MM, Devi TM, Sarangthem B, Keisham S, Devi PM. Prevalence of tuberculosis: A study in forensic autopsies. J Med Soc 2014;28:162-5

5. Joaquim AF, Carandina L, Defaveri J. Tuberculose em necropsias realizadas no Serviço de Anatomia Patológica da Faculdade de Medicina de Botucatu. J Bras Patol Med Lab, 2006;42(3):193-200. 
6. Flavin RJ, Gibbons N, O’BRIAIN DS. Mycobacterium tuberculosis at autopsy - exposure and protection: an old adversary revisited. J Clin Pathol. 2007;60(5):487-91.

7. Garg M, Aggarwal AD, Singh S, Kataria SP. Tuberculous Lesions at Autopsy. J Indian Acad Forensic Med. 2011;33(2):116-9.

8. Chaves J, Tomilin BA, Brun D, Fuhr LG, Ourique F, Pilletti K, et al. Perfil dos pacientes com tuberculose que foram atendidos em uma unidade de referênica do município de Santa Cruz do Sul - RS no período de 2009 a 2013. J Health Biol Sci. 2017;5(1):31-6.

9. Chihana M, Floyd S, Molesworth A, Crampin AC, Kayuni N, Price A, et al. Adult mortality and probable cause of death in rural northern Malawi in the era of HIV treatment. Trop Med Int Health. 2012;17(8):74- 83 .

10. CARRAZEDO AM. Aspectos distintos da Tuberculose em imunocompetentes e imunocomprometidos: epidemiologia, manifestações clínicas, exames auxiliares de diagnóstico e tratamento [Internet]. Porto: Universidade do Porto; 2009 [cited 2020 Oct. 06]. Available from: https://repositorio-aberto.
up.pt/bitstream/10216/20894/2/Aspectos\%20distintos\%20 da $\% 20$ Tuberculose $\% 20 \mathrm{em} \% 20$ imunocompetentes $\% 20 \mathrm{e} \% 20$ imunocomprometido\%20epidemiologia\%20manifest.pdf

11. Silva DR, Munoz-Torrico M, Duarte R, Galvão T, Bonini EH, Arbex FF, et al. Fatores de risco para tuberculose: diabetes, tabagismo, álcool e uso de outras drogas. J Bras Pneumol. 2018;44(2):145-52.

12. Sharma SK, Mohan A, Sharma A. Miliary tuberculosis: A new look at an old foe. J Clin Tuberc Other Mycobact Dis. 2016;3:13-27.

13. Suarez I, Funger SM, Kroger S, Rademacher J, Fatkenheuer G, Rybniker J. The Diagnosis and Treatment of Tuberculosis. Dtsch Arztebl Int. 2019 Oct 25;116(43):729-35.

14. Bavikar R, Valand A, Kumar H, Gore C, Iqbal MB. Tuberculosis at autopsy in inpatients at a tertiary referral centre in India: a prospective study. Indian J Pathol Oncol 2015;2(4):186-8 .

15. Khusro A, Aarti C. Extrapulmonary tuberculosis: An overview on infection beyond Lungs. World News of Natural Sciences. 2020;18:131-41

\section{Como citar:}

Feitosa PM, Ferreira MV, Silveira RS, Queiroz TS, Aguiar LF, Carvalho LN. Tuberculose em 228 casos de autópsias. Rev Med UFC. 2021;61(1):1-5. 